\title{
QUASISYMMETRIC AND UNIPOTENT TENSOR CATEGORIES
}

\author{
Pavel Etingof and Shlomo Gelaki
}

\section{Introduction}

One of the most important early developments in the theory of quantum groups was Drinfeld's classification, in characteristic zero, of quasitriangular quasi-Hopf QUE (quantized universal enveloping) algebras [Dr1, Dr2]. In the language of tensor categories, this is, in essence, a classification of 1-parameter flat formal deformations, as a braided category ${ }^{1}$, of the representation category of a Lie algebra ${ }^{2} \mathfrak{g}_{0}$. The answer is that such deformations are parameterized by pairs $(\mathfrak{g}, t)$, where $\mathfrak{g}$ is a flat formal deformation of $\mathfrak{g}_{0}$, and $t$ is an element in $\left(S^{2} \mathfrak{g}\right)^{\mathfrak{g}}$. More specifically, the deformed category is the category of representations of $\mathfrak{g}$, with the usual tensor product functor, the braiding is given by the formula $\beta=P \circ e^{\hbar t / 2}$, where $P$ is the flip map, and the associativity isomorphism is $\Phi\left(\hbar t_{12}, \hbar t_{23}\right)$, where $\Phi(a, b)$ is any Drinfeld associator. In particular, symmetric deformations correspond to the case $t=0$; in other words, such deformations come simply from deformations $\mathfrak{g}$ of the corresponding Lie algebra $\mathfrak{g}_{0}$.

Drinfeld's result generalizes mutatis mutandis to a more general setting where $\mathfrak{g}_{0}$ is a Lie superalgebra, and to the situation when $\mathfrak{g}_{0}$ is replaced with an affine proalgebraic supergroup $G_{0}$. More specifically, suppose that $G_{0}$ is an affine proalgebraic supergroup, and $u_{0} \in G_{0}$ an element of order 2 acting by parity on the function algebra $\mathcal{O}\left(G_{0}\right)$. Let $\operatorname{Rep}\left(G_{0}, u_{0}\right)$ be the category of representations of $G_{0}$ on finite dimensional supervector spaces, in which $u_{0}$ acts by parity. Then Drinfeld's work implies that any flat formal deformation of the category $\operatorname{Rep}\left(G_{0}, u_{0}\right)$, as a braided category, has the form $\operatorname{Rep}(G, u)$, where $G$ is a deformation of $G_{0}, u$ is the (unique) deformation of $u_{0}$ in $G$, and the associativity isomorphism and braiding are $\Phi\left(\hbar t_{12}, \hbar t_{23}\right)$ and $P \circ e^{\hbar t / 2}$, for some $t \in\left(S^{2} \mathfrak{g}\right)^{G}$ (here $\mathfrak{g}=\operatorname{Lie}(G)$ ). Moreover, $(G, u, t)$ are determined by the deformation uniquely up to an isomorphism. In particular, if the deformation is symmetric then $t=0$, and the deformation is $\operatorname{Rep}(G, u)$.

By Deligne's theorem [De2], any symmetric tensor category of exponential growth has the form $\operatorname{Rep}\left(G_{0}, u_{0}\right)$; thus, Drinfeld's result provides a description of flat formal deformations of any symmetric tensor category with at most exponential growth. In particular, in the special case of symmetric deformations, Drinfeld's result can be viewed as a formal analog of Deligne's theorem.

\footnotetext{
Received by the editors August 10, 2007.

${ }^{1}$ Here by a flat formal deformation of the category of finite dimensional modules over a (topological) algebra $A_{0}$ we mean the category of finite dimensional modules over a flat formal deformation $A$ of $A_{0}$. This definition will suffice for our purposes; we note, however, that in general, the definition of a flat formal deformation of an abelian category is fairly nontrivial ([LV]).

${ }^{2}$ Throughout the paper, we work over the ground field $\mathbb{C}$ of complex numbers.
} 
Unfortunately, Drinfeld's method makes a serious use of the presence of the formal parameter $\hbar$, i.e., of the fact that the braided categories at hand are symmetric modulo this parameter. For this reason, it cannot be applied to classifying braided categories over $\mathbb{C}$, not involving $\hbar$ (even those of exponential growth). In fact, we are very far from the classification of such categories, even in the special case of finite semisimple (i.e., fusion) categories.

On the other hand, it turns out that there is a subclass of braided categories for which Drinfeld's method does work. This is the class of quasisymmetric categories, introduced essentially in $[\mathrm{EK} 2]^{3}$. They are, by definition, braided categories with exponential growth, in which the square of the braiding is the identity on the product of any two simple objects. For such categories, the infinite power series in $\hbar$ which occur in Drinfeld's construction terminate when applied to the tensor product of any objects, and thus become polynomials in $\hbar$. These polynomials can then be evaluated at $\hbar=1$, which allows one to apply Drinfeld's method to the situation without $\hbar$.

The study of quasisymmetric categories by means of Drinfeld's method is one of the main goals of this paper. Another is to study unipotent tensor categories, i.e., such that every simple object is the neutral object. Namely, in Section 2, using Drinfeld's method and Deligne's theorem, we give a classification of quasisymmetric categories, which is similar to Drinfeld's classification of quasitriangular quasi-Hopf QUE algebras. Specifically, we show that equivalence classes of such categories are in bijection with equivalence classes of triples $(G, u, t)$, where $G$ is an affine proalgebraic supergroup, $u \in G$ an element of order 2 which acts by parity on the function algebra $\mathcal{O}(G)$, and $t$ a nilpotent element of $\left(S^{2} \mathfrak{g}\right)^{G}$, where $\mathfrak{g}=\operatorname{Lie}(G)$. This gives a generalization of Deligne's theorem to the case of quasisymmetric categories. As a special case, this result yields a classification of braided unipotent categories. In Section 3 , we proceed to classify unipotent fiber functors on quasisymmetric categories, i.e., functors that are "standard" on the canonical symmetric part of the category (the subcategory $\otimes$-generated by the simple objects). Namely, we show that such functors are in bijection with nilpotent solutions $r$ of the classical Yang-Baxter equation, such that $r+r^{21}=t$. In Section 4, using the quantization theory from [EK1, EK2, EK3], we classify coconnected Hopf algebras (i.e., Hopf algebras with a unique simple comodule) by showing that they all come from quantization of prounipotent Poisson proalgebraic groups. This provides a classification of unipotent tensor categories with a fiber functor.

Acknowledgments. The research of the first author was partially supported by the NSF grant DMS-0504847. The second author was supported by The Israel Science Foundation (grant No. 125/05). He also thanks MIT for its warm hospitality during his Sabbatical. Both authors were supported by BSF grant No. 2002040.

\section{Quasisymmetric categories}

Let $\mathcal{C}$ be a rigid tensor category over $\mathbb{C}$. In particular, $\mathcal{C}$ is an abelian category, with finite dimensional spaces of morphisms and all objects having finite length, and we have $\operatorname{End}(\mathbf{1})=\mathbb{C}$. We will also assume throughout the paper that $\mathcal{C}$ has exponential

\footnotetext{
${ }^{3}$ To be precise, the definition of a quasisymmetric category in [EK2] is somewhat different from the one used in this paper, but the difference is inessential for what we do.
} 
growth, i.e., that for every object $Y$ there exists $d(Y) \geq 1$ such that $\operatorname{length}\left(Y^{\otimes n}\right) \leq$ $d(Y)^{n}$ for all $n \geq 1$.

\subsection{Unipotent categories.}

Definition 2.1. The tensor category $\mathcal{C}$ is called unipotent if the only simple object in $\mathcal{C}$ is the neutral object $\mathbf{1}$.

Remark 2.2. Note that the exponential growth condition is automatic for unipotent tensor categories.

The simplest example is the following one.

Example 2.3. Let $G$ be a prounipotent proalgebraic group. Then $\mathcal{C}:=\operatorname{Rep}(G)$, the category of rational representations of $G$, is unipotent.

Proposition 2.4. If $\mathcal{C}$ is a symmetric unipotent category then $\mathcal{C}=\operatorname{Rep}(G)$ for some prounipotent proalgebraic group $G$.

Proof. This follows from Deligne's theorem [De1] since the categorical dimension of an object $X$ is just its length, hence a positive integer.

2.2. Unipotent radical of a supergroup. Let $G$ be an affine proalgebraic supergroup ${ }^{4}$. Denote by $U \subset G$ the intersection of the kernels of irreducible algebraic representations of $G$, and set $G_{\text {red }}:=G / U$.

Thus, we have a natural exact sequence of supergroups

$$
1 \rightarrow U \rightarrow G \rightarrow G_{\text {red }} \rightarrow 1 \text {. }
$$

Definition 2.5. We will call the supergroup $G_{\text {red }}$ the reductive quotient of $G$, and $U$ the unipotent radical of $G$. We will say that a supergroup $G$ is reductive if $U=1$ and $G=G_{\text {red }}$.

Note that for a reductive supergroup, it is not always true that the category of its representations is semisimple: for example, the supergroup $G L(m \mid n)$ is reductive, but it has representations which are reducible but indecomposable.

2.3. Quasisymmetric categories. Let $\mathcal{C}$ be a braided category with braiding $\beta$.

Definition 2.6. Let us say that $\mathcal{C}$ is quasisymmetric if for every simple objects $X, Y \in \mathcal{C}$, one has $\beta^{2}=\operatorname{Id}$ on $X \otimes Y$.

Example 2.7. Every symmetric and every unipotent tensor category is quasisymmetric.

Let $\mathcal{C}$ be a quasisymmetric tensor category. Denote by $\mathcal{C}_{s}$ the full tensor subcategory of $\mathcal{C} \otimes$-generated by simple objects of $\mathcal{C}$ (i.e., formed by the subquotients of direct sums of tensor products of simple objects).

The following proposition is obvious from the braiding axioms.

Proposition 2.8. The category $\mathcal{C}_{s}$ is symmetric.

Definition 2.9. We will call $\mathcal{C}_{s}$ the canonical symmetric part of $\mathcal{C}$.

\footnotetext{
${ }^{4}$ For a short introduction to supergroups and Deligne's theorem [De2] we refer the reader, for example, to [EG2].
} 
Remark 2.10. Note that a quasisymmetric category $\mathcal{C}$ is unipotent if and only if $\mathcal{C}_{s}$ is the category of finite dimensional vector spaces.

Example 2.11. Let $G$ be an affine proalgebraic supergroup, and $u \in G$ an element of order 2 acting by parity on the algebra of regular functions $\mathcal{O}(G)$, and let $\operatorname{Rep}(G, u)$ be the category of representations of $G$ on finite dimensional supervector spaces on which $u$ acts by parity. Then $\mathcal{C}_{s}=\operatorname{Rep}\left(G_{\text {red }}, u\right)$.

By Deligne's theorem [De2], Proposition 2.8 implies the following corollary.

Corollary 2.12. Let $\mathcal{C}$ be a quasisymmetric category. There exists a unique pair $\left(G_{\mathrm{red}}, u\right)$, where $G_{\mathrm{red}}$ is a reductive proalgebraic supergroup, and $u \in G_{\mathrm{red}}$ an element of order 2 acting by parity on $\mathcal{O}\left(G_{\text {red }}\right)$, such that $\mathcal{C}_{s}=\operatorname{Rep}\left(G_{\mathrm{red}}, u\right)$.

2.4. Construction of quasisymmetric categories. Recall that for an affine proalgebraic supergroup $G$, its Lie (super)algebra $\operatorname{Lie}(G)$ is defined as the set of left invariant derivations of $\mathcal{O}(G)$.

Let $G$ be an affine proalgebraic supergroup with Lie algebra $\mathfrak{g}$. Let $G_{\text {red }}$ be the reductive quotient of $G, U$ the unipotent radical of $G$, and $\mathfrak{g}_{r}, \mathfrak{u}$ their Lie algebras.

Definition 2.13. Let us say that an element of the tensor square $\mathfrak{g}^{\otimes 2}$ is nilpotent if it projects to zero in $\mathfrak{g}_{r}^{\otimes 2}$, i.e., if $t \in \mathfrak{g} \otimes \mathfrak{u}+\mathfrak{u} \otimes \mathfrak{g}$.

Let $u \in G$ be an element of order 2 acting on $\mathcal{O}(G)$ by parity. Let $t \in\left(S^{2} \mathfrak{g}\right)^{G}$ be an invariant nilpotent symmetric 2 -tensor.

Let $\Phi=\Phi(a, b)$ be a Lie associator of Drinfeld; it is an element in the completed free associative algebra in two non-commuting variables $a, b$ satisfying some equations [Dr2] (see also [ES], page 158).

Now let $\mathcal{C}(G, u, t, \Phi)$ be the braided tensor category defined as follows. As an abelian category it is just $\operatorname{Rep}(G, u)$. The tensor product bifunctor is the usual one, while the associativity constraint is given by $\alpha:=\Phi\left(t_{12}, t_{23}\right)$ (i.e., $\alpha_{\mid X \otimes Y \otimes Z}=$ $\left.\Phi\left(t_{12}, t_{23}\right)_{\mid X \otimes Y \otimes Z}\right)$. The braiding is given by $\beta:=P \circ e^{t / 2}$, where $P$ is the standard flip map.

Remark 2.14. Let us explain why $\alpha$ is well defined (the explanation for $\beta$ is similar). Recall that any object $X$ in a finite length abelian category has a canonical filtration $F^{\bullet}: F^{0}(X)$ is the sum of all simple subobjects of $X$, and $F^{i}(X)$ is defined inductively as the preimage in $X$ of $F^{i-1}\left(X / F^{0}(X)\right)$. It is clear that if $\mathcal{C}=\operatorname{Rep}(G, u)$, and $a \in \mathfrak{u}$, then $a F^{i}(X) \subset F^{i-1}(X)$, so $a$ lowers the filtration degree by 1 . This implies that if the lengths of $X, Y, Z$ are $l_{X}, l_{Y}, l_{Z}$, and $n>l_{X}+l_{Y}+l_{Z}-3$, then any product $t_{i_{1} j_{1} \ldots t_{i_{n} j_{n}}}$ acts by zero in $X \otimes Y \otimes Z$. This means that the series defining $\alpha$ terminates, and thus $\alpha$ is well defined.

Proposition 2.15. We have

(i) The category $\mathcal{C}(G, u, t, \Phi)$ is quasisymmetric.

(ii) For all $\Phi, \Phi^{\prime}, \mathcal{C}(G, u, t, \Phi)$ is equivalent to $\mathcal{C}\left(G, u, t, \Phi^{\prime}\right)$.

\footnotetext{
${ }^{5}$ Since $G$ is a affine proalgebraic group, $\mathfrak{g}$ is a provector space. Thus tensor powers of $\mathfrak{g}$ will be understood in the completed sense.
} 
Proof. (i) Since $t$ acts by zero in $X \otimes Y$, where $X$ and $Y$ are simple $G$-modules, the result follows.

(ii) Set $\Phi_{1}:=\Phi\left(\hbar t_{12}, \hbar t_{23}\right), \Phi_{2}:=\Phi^{\prime}\left(\hbar t_{12}, \hbar t_{23}\right)$. By Theorem 3.15 in [Dr1], there exists an invariant symmetric twist $T:=T(\hbar t) \in\left(U(\mathfrak{g})^{\otimes 2}\right)^{G}[[\hbar]]$, given by a universal formula, such that $\Phi_{1}^{T}=\Phi_{2}$. Now, since $t$ is nilpotent, similarly to Remark 2.14, $T(\hbar t)_{\mid X \otimes Y}$ is a polynomial in $\hbar$ for all $X, Y$, and hence can be evaluated at $\hbar=1$. So $T(t)$ is a well defined functorial morphism $X \otimes Y \rightarrow X \otimes Y$. The identity functor $\mathcal{C}(G, u, t, \Phi) \rightarrow \mathcal{C}\left(G, u, t, \Phi^{\prime}\right)$ equipped with the tensor structure $T(t)$ is an equivalence of braided tensor categories, as desired.

Remark 2.16. By Proposition 2.15, we may (and will) denote $\mathcal{C}(G, u, t, \Phi)$ simply by $\mathcal{C}(G, u, t)$. Note that $\mathcal{C}(G, u, 0)=\operatorname{Rep}(G, u)$ as a braided tensor category. If $u=1$, we will write $\mathcal{C}(G, t)$ for $\mathcal{C}(G, 1, t)$.

2.5. Classification of quasisymmetric categories. Let $\overline{G T}=\overline{G T}(\mathbb{C})$ be the Grothendieck-Teichmüller semigroup, defined by Drinfeld [Dr2]. Recall that this semigroup consists of pairs $(\lambda, f)$, where $\lambda \in \mathbb{C}$ and $f(A, B)=e^{\tilde{f}(\log A, \log B)}$, where $\tilde{f}$ is a formal Lie series satisfying some properties.

Recall (see e.g. [EK2], Section 2.2) that the semigroup $\overline{G T}$ acts on the set of equivalence classes of quasisymmetric categories. Namely, let $\mathcal{C}$ be a quasisymmetric category. The action of $g=(\lambda, f)$ on $\mathcal{C}$ is given by preserving the abelian category structure and the functor of tensor product, and transforming the associativity isomorphism and braiding by the formulas

$$
\begin{gathered}
\beta^{\prime}=\beta \circ\left(\beta^{2}\right)^{\frac{\lambda-1}{2}}, \\
\alpha^{\prime}=\alpha \circ f\left(\beta_{12}^{2}, \alpha^{-1} \circ \beta_{23}^{2} \circ \alpha\right) .
\end{gathered}
$$

Remark 2.17. Note that $\beta^{\prime}$ in the above formula is well defined since $\beta^{2}-1$ is nilpotent. Namely, for any complex number $s,\left(\beta^{2}\right)^{s}$ is by definition equal to $e^{s \log \beta^{2}}$, where

$$
\log \beta^{2}=\log \left(1+\left(\beta^{2}-1\right)\right)=\sum_{m \geq 1}(-1)^{m-1} \frac{\left(\beta^{2}-1\right)^{m}}{m} .
$$

Recall also ([Dr2], Proposition 5.2) that every Lie associator $\Phi$ gives rise to a canonical 1-parameter subsemigroup $g_{\Phi}(\lambda)=\left(\lambda, f_{\Phi}(\lambda)\right)$.

We can now state our first main result.

Theorem 2.18. (i) Any quasisymmetric tensor category is equivalent, as a braided tensor category, to $\mathcal{C}(G, u, t)$ for some $(G, u, t)$ with nilpotent $t \in\left(S^{2} \mathfrak{g}\right)^{G}$.

(ii) $\mathcal{C}(G, u, t)$ is equivalent to $\mathcal{C}\left(G^{\prime}, u^{\prime}, t^{\prime}\right)$ if and only if there exists a supergroup isomorphism $\phi: G \rightarrow G^{\prime}$ sending $u$ to $u^{\prime}$ such that $(d \phi \otimes d \phi)(t)=t^{\prime}$.

(iii) Any braided unipotent tensor category is equivalent, as a braided tensor category, to $\mathcal{C}(G, t)$, where $G$ is a prounipotent proalgebraic group, and $t \in\left(S^{2} \mathfrak{g}\right)^{G}$. The pair $(G, t)$ is determined uniquely up to an isomorphism.

Proof. Let $\mathcal{C}$ be a quasisymmetric category. Consider the 1-parameter family of quasisymmetric categories $\mathcal{C}(\lambda):=g_{\Phi}(\lambda)(\mathcal{C})($ so $\mathcal{C}(1)=\mathcal{C}$ ). This family depends polynomially on $\lambda$ : the abelian category structure and the tensor product functor do not 
change with $\lambda$, while the associativity isomorphism and the braiding depend polynomially on $\lambda$.

The main point is that the category $\mathcal{C}(0)$ is symmetric. Therefore, by Deligne's theorem [De2], it is equivalent to $\operatorname{Rep}(G, u)$ for some $(G, u)$. Thus we can identify $\mathcal{C}$ with $\operatorname{Rep}(G, u)$ as an abelian category with the tensor product functor. Then the category $\mathcal{C}(\lambda)$ can be described as follows. The braiding in this category is $P \circ$ $e^{\lambda t / 2}$, where $P$ is the symmetry morphism of $\operatorname{Rep}(G, u)$ and $t=\log \left(\beta^{2}\right)$, and the associativity isomorphism is $\alpha=\Phi\left(\lambda t_{12}, \lambda t_{23}\right)$. Expanding the hexagon relations in powers of $\lambda$ and taking the linear part, we find that $t_{12,3}=t_{13}+t_{23}$, which implies that $t \in\left(S^{2} \mathfrak{g}\right)^{G}$. Moreover, because of the quasisymmetry condition, $t$ is nilpotent. Thus, setting $\lambda=1$, we get $\mathcal{C}=\mathcal{C}(G, u, t, \Phi)=\mathcal{C}(G, u, t)$. This proves part (i) of the theorem.

To prove part (ii), assume that we have an equivalence of braided tensor categories $F: \mathcal{C}(G, u, t, \Phi) \rightarrow \mathcal{C}\left(G^{\prime}, u^{\prime}, t^{\prime}, \Phi\right)$. Applying the semigroup $g_{\Phi}(\lambda)$ to this equivalence, we get an equivalence $F_{\lambda}: \mathcal{C}(G, u, \lambda t, \Phi) \rightarrow \mathcal{C}\left(G^{\prime}, u^{\prime}, \lambda t^{\prime}, \Phi\right)$. By setting $\lambda=0$ and using Deligne's theorem, we can assume that $(G, u)=\left(G^{\prime}, u^{\prime}\right)$ and $F$ is the identity functor. Then we get $t=t^{\prime}$, as desired. This proves (ii).

Part (iii) follows from (i) and (ii).

This completes the proof of the theorem.

\section{Fiber functors on quasisymmetric categories}

3.1. Unipotent fiber functors. Let $\mathcal{C}$ be a quasisymmetric category (so $\mathcal{C}=$ $\mathcal{C}(G, u, t)$, where $t$ is nilpotent).

Definition 3.1. Let us say that a fiber functor $F: \mathcal{C} \rightarrow$ Vec is unipotent if it coincides with the standard one on the subcategory $\mathcal{C}_{s}=\operatorname{Rep}\left(G_{\text {red }}, u\right)$.

Let us give a construction of unipotent fiber functors. (In the case $t=0$, this construction appears in [EG1], Theorem 5.5.) Let $r \in \mathfrak{g} \otimes \mathfrak{g}$ be a nilpotent solution to the classical Yang-Baxter equation

$$
\left[r_{12}, r_{13}\right]+\left[r_{12}, r_{23}\right]+\left[r_{13}, r_{23}\right]=0,
$$

such that $t=r+r_{12}$. Then by the results of [EK1], [EK2], [E], there exists a universal formula $J=J(\hbar r)$ defining a pseudotwist killing the associator $\Phi\left(\hbar t_{12}, \hbar t_{23}\right)$. Since $r$ is nilpotent, similarly to Remark 2.14 , the series $J(\hbar r)$, when evaluated in any product $X \otimes Y$, is in fact a polynomial in $\hbar$. Thus, it can be evaluated at $\hbar=1$. This gives rise to a unipotent fiber functor $F_{r}: \mathcal{C} \rightarrow$ Vec, which is the usual forgetful functor on $\operatorname{Rep}(G, u)$ with tensor structure defined by $J(r)$.

3.2. Classification of unipotent fiber functors. Our second main result is the following one.

Theorem 3.2. If $F$ is a unipotent fiber functor on $\mathcal{C}$ then $F=F_{r}$ for some nilpotent $r$, and $r$ is uniquely determined up to conjugation.

Proof. As we have already mentioned above, according to [EK1, EK2, E], the quantization $\left(U_{\hbar}(\mathfrak{g}), R\right)$ of a quasitriangular Lie bialgebra $(\mathfrak{g}, r)$ can be obtained by twisting 
the enveloping algebra $U(\mathfrak{g})[[\hbar]]$ by a pseudotwist $J(\hbar r)$, given by a certain universal formula. The quantum $R$-matrix of $U_{\hbar}(\mathfrak{g})$ is then given by the universal formula

$$
R(\hbar r)=J_{21}(\hbar r) e^{\hbar\left(r+r_{21}\right) / 2} J(\hbar r)^{-1}=1+\hbar r+O\left(\hbar^{2}\right) .
$$

Thus

$$
\log R=H(\hbar r)=\hbar r+O\left(\hbar^{2}\right),
$$

where $H$ is some infinite series. Since $H$ is the identity modulo higher terms, this formula can be inverted:

$$
\hbar r=H^{-1}(\log R) .
$$

Now suppose that $F$ is a unipotent fiber functor on $\mathcal{C}$. Then $B=\operatorname{End}(F)$ is a (topological) quasitriangular Hopf algebra. Let us now apply formula (1) to the R-matrix of $B, R=P \circ F(\beta)$. Because of the unipotency of $F$, this R-matrix is unipotent, and hence $H^{-1}(\log R)$ makes sense, even though $H^{-1}$ is an infinite series, and there is no formal parameter $\hbar$. So we can set $r=H^{-1}(\log R)$. Let us twist $B$ by the twist $J(r)^{-1}$, and denote the corresponding Hopf algebra by $B_{0}$. It follows from the fact that the quantization of quasitriangular Lie bialgebras gives rise to a prop isomorphism (Section 5 of [EE]) that $B_{0}$ is cocommutative, and the category $\operatorname{Rep}\left(B_{0}\right)$ with trivial symmetric structure is equivalent to $\operatorname{Rep}(G, u)$ as a symmetric tensor category. Moreover, $r \in \mathfrak{g} \otimes \mathfrak{g}$, and $B_{0}$ is equipped with a quasitriangular co-Poisson structure defined by $r$. It is now clear that $F=F_{r}$. This proves the existence part of the theorem. The uniqueness of $r$ up to conjugation follows from the canonicity of the above construction (see also Theorem 5.3 of [EE]).

Remark 3.3. Note that if $\mathcal{C}$ is a unipotent category, then the conditions of unipotency of $F$ and nilpotency of $r$ are vacuous and can be dropped.

Corollary 3.4. Let $\mathcal{C}=\operatorname{Rep}(G, t)$, where $G$ is a prounipotent proalgebraic group. Then the assignment $r \rightarrow F_{r}$ defines a bijection between isomorphism classes of fiber functors on $\mathcal{C}$ and elements $r \in \mathfrak{g} \otimes \mathfrak{g}$ which satisfy the classical Yang-Baxter equation and the condition $r+r^{21}=t$.

Note that if $G$ is a unipotent algebraic group, and $r$ is as in Corollary 3.4 with $t=0$ (i.e., $r \in \wedge^{2} \mathfrak{g}$ ) then by a well known theorem of Drinfeld (see [ES]), the image of $r$ (regarded as a map $\mathfrak{g}^{*} \rightarrow \mathfrak{g}$ ) is a Lie subalgebra $\mathfrak{h} \subset \mathfrak{g}$ defining a closed subgroup $H \subset G$, and $\omega=r^{-1}$ is a nondegenerate 2-cocycle (i.e., a left-invariant symplectic form) on $H$. Conversely, for any closed subgroup $H \subset G$ and a leftinvariant symplectic form $\omega$ on $H$, the element $r=\omega^{-1}$, regarded as an element of $\wedge^{2} \mathfrak{g}$, is a solution of the classical Yang-Baxter equation. Thus, Corollary 3.4 implies

Corollary 3.5. Let $\mathcal{C}=\operatorname{Rep}(G)$, where $G$ is a unipotent algebraic group. Then equivalence classes of fiber functors on $\mathcal{C}$ are in bijection with conjugacy classes of pairs $(H, \omega)$, where $H$ is a closed subgroup of $G$, and $\omega$ is a left invariant symplectic form on $H$.

This corollary is an analog, for unipotent algebraic groups, of the classification of fiber functors on $\operatorname{Rep}(G)$ for finite group $G$, due to Movshev [M]. 
Remark 3.6. We note that a classification of fiber functors on $\operatorname{Rep}(G, u)$ for a general affine algebraic supergroup $G$ is unknown. It is clear that to obtain such a classification, it would be sufficient to do so for $G=G L(m \mid n)$, which is equivalent to classifying unitary solutions of the quantum Yang-Baxter equation which are invertible and skew-invertible. This problem is open even for $n=0$ (starting from $m=4)$.

\subsection{Classification of coconnected coquasitriangular Hopf algebras.}

Definition 3.7. (see e.g. $[\mathrm{S}]$ ) A Hopf algebra $A$ is called coconnected if every simple comodule over $A$ is trivial.

Corollary 3.8. Let $A$ be a coconnected coquasitriangular Hopf algebra. Then A is obtained by twisting the product of the function algebra $\mathcal{O}(G)$ of a prounipotent proalgebraic group $G$ by a pseudotwist $J=J(r)$, where $r \in \mathfrak{g}^{\otimes 2}$ is a solution of the classical Yang-Baxter equation, such that $t=r+r^{21}$ is $G$-invariant. Moreover, the pair $(G, r)$ is determined by $A$ up to an isomorphism.

Proof. Follows from Theorem 2.18(iii) and Corollary 3.4, since the comodule category of a coconnected coquasitriangular Hopf algebra is a unipotent braided tensor category.

\section{Classification of coconnected Hopf algebras}

In this section we will give a classification of coconnected Hopf algebras.

4.1. Construction of coconnected Hopf algebras. Let $G$ be a prounipotent Poisson proalgebraic group, and $\mathfrak{g}$ its Lie algebra. Then $\mathfrak{g}$ has a Lie bialgebra structure $\delta$ which determines the Poisson-Lie structure on $G$. Consider the lower central series filtration $\mathfrak{g}$ • on $\mathfrak{g}: \mathfrak{g}_{0}=\mathfrak{g}$ and $\mathfrak{g}_{i+1}=\left[\mathfrak{g}_{i}, \mathfrak{g}\right]$. Then the bracket [,] of $\mathfrak{g}$ has degree 1 with respect to this filtration, while the cobracket $\delta$ has degree 0 (since $\delta([a, b])=$ $[a \otimes 1+1 \otimes a, \delta(b)]+[\delta(a), b \otimes 1+1 \otimes b])$.

Consider now the Etingof-Kazhdan quantization $\mathcal{O}_{\hbar}(G)$ of the Poisson group $G$, [EK3]. This is the space $\mathcal{O}(G)[[\hbar]]$ with certain product and coproduct deforming the standard Hopf algebra structure on $\mathcal{O}(G)$. These product and coproduct are given by some universal formulas (infinite series) in terms of the bracket and cobracket of $\mathfrak{g}$. Because the degree of the bracket is 1 and of the cobracket is zero, these infinite series terminate, and thus the formal quantization of $G$ is actually defined over the polynomials $\mathbb{C}[\hbar]$, which means that the Hopf algebra $\mathcal{O}_{\hbar}(G)$ has a lattice $\mathcal{O}_{\hbar}(G)_{\text {pol }}$ over $\mathbb{C}[\hbar]$. Specializing this lattice to $\hbar=1$, we get a Hopf algebra $A=A(G, \delta)$ over C. As a vector space, it coincides with $\mathcal{O}(G)$.

Lemma 4.1. The Hopf algebra $A$ is coconnected.

Proof. Let $A_{0}:=\mathcal{O}(G)$, and let $C_{\bullet}\left(A_{0}\right)$ be the coradical filtration of $A_{0}$. Since $A_{0}$ is coconnected, $C_{0}\left(A_{0}\right)=\mathbb{C}$. It is easy to see that the product in $A_{0}$ preserves this filtration, while the Poisson bracket preserves it in the strict sense, i.e., decreases the filtration degree by 1 . This implies that the product and coproduct in $A$ preserve the coradical filtration (as they are obtained from the product, coproduct, and Poisson bracket of $A_{0}$ by a universal formula). In particular, the coradical filtration $C \cdot(A)$ of $A$ coincides with $C_{\bullet}\left(A_{0}\right)$. This implies that $A$ is coconnected. 
4.2. Classification of coconnected Hopf algebras. Our third main result is the following theorem.

Theorem 4.2. Any coconnected Hopf algebra over $\mathbb{C}$ is of the form $A(G, \delta)$, and $(G, \delta)$ is determined up to an isomorphism.

Proof. The results of [EK2],[EE] imply that the formulas expressing the product $\mu$ and coproduct $\Delta$ of $\mathcal{O}_{\hbar}(G)$ in terms of the product $\mu_{0}$, coproduct $\Delta_{0}$, and bracket $\delta$ of $\mathcal{O}(G)$ are invertible. Let $A$ be a coconnected Hopf algebra, and let us apply the inverse formulas at $\hbar=1$ to introduce a commutative product $\mu_{0}$, coproduct $\Delta_{0}$, and bracket $\delta$ on $A$. As before, the formulas make sense for $\hbar=1$ because series terminate due to the pronilpotency of $\mathfrak{g}$. In this way we get a commutative Poisson-Hopf algebra $A_{0}$, which coincides with $A$ as a vector space.

Let $C_{\bullet}(A)$ be the coradical filtration of $A$. Since $A$ is coconnected, $C_{0}(A)=\mathbb{C}$. Also, this filtration is fixed by the product and coproduct $\mu, \Delta$ in $A$. Hence it is fixed by the new coproduct $\Delta_{0}$, as $\Delta_{0}$ expresses via $\mu, \Delta$ by a universal formula. Thus $C \bullet(A)$ coincides with the coradical filtration $C \bullet\left(A_{0}\right)$ of $A_{0}$. Hence $A_{0}$ is coconnected, i.e., $A_{0}=\mathcal{O}(G)$. It is now clear that $A=A(G, \delta)$. The uniqueness of $G, \delta$ is clear from the canonicity of this construction. The theorem is proved.

Remark 4.3. A classical theorem of Kostant states that a coconnected cocommutative Hopf algebra over $\mathbb{C}$ is the same thing as an enveloping algebra of a Lie algebra. This fits with Theorem 4.2 as follows. It is easy to show that the cocommutativity condition of $A(G, \delta)$ is equivalent to the condition that $G$ is abelian. Thus $G=\mathfrak{g}$ is a provector space. Denote the topological dual space $\mathfrak{g}^{*}$ by $L$; it is an ordinary (discrete) vector space, possibly infinite dimensional. Then $\delta$ gives rise to a Lie bracket on $L$, and it is easy to see that $A(G, \delta)=U(L)$.

Remark 4.4. In categorical terms, Theorem 4.2 provides a classification of unipotent tensor categories with a fiber functor; namely, they are categories of finite dimensional comodules over Hopf algebras $A(G, \delta)$.

Remark 4.5. If $\mathcal{C}$ is a finite unipotent tensor category, then it is shown in [EO], Section 2.10, that $\mathcal{C}$ is the category of vector spaces. This is a special case of Theorem 4.2 , since in the finite case we must have $G=1$.

Remark 4.6. One may hope that along these lines one should be able to obtain a classification of general unipotent tensor categories; namely, one could expect that their equivalence classes are in a natural bijection with equivalence classes of prounipotent groups $G$ with a Lie quasibialgebra structure on $\operatorname{Lie}(G)$. Unfortunately, this remains out of reach, as a quantization theory of Lie quasibialgebras is still unavailable.

Remark 4.7. We have obtained several theorems giving a classification of various kinds of tensor categories in terms of affine proalgebraic supergroups with some additional data. We note that in these theorems, an additional requirement that the relevant affine proalgebraic supergroup be actually algebraic is equivalent to the requirement that the corresponding category $\mathcal{C}$ be finitely $\otimes$-generated $([\mathrm{De} 2])$, i.e., there exists an object $X$ in $\mathcal{C}$ such that every object in $\mathcal{C}$ is a subquotient of a direct sum of tensor powers of $X$. 


\section{References}

[De1] P. Deligne, Categories Tannakiennes, In The Grothendick Festschrift, Vol. II, Prog. Math. 87 (1990), 111-195.

[De2] Catégories tensorielles. (French) [Tensor categories] Dedicated to Yuri I. Manin on the occasion of his 65th birthday. Mosc. Math. J. 2 (2002), no. 2, 227-248.

[Dr1] V. Drinfeld, Quasi-Hopf algebras. (Russian) Algebra i Analiz 1 (1989), no. 6, 114-148; translation in Leningrad Math. J. 1 (1990), no. 6, 1419-1457.

[Dr2] - On quasitriangular quasi-Hopf algebras and on a group that is closely connected with $\operatorname{Gal}(\overline{\mathbb{Q}} / \mathbb{Q})$. (Russian) Algebra i Analiz 2 (1990), no. 4, 149-181; translation in Leningrad Math. J. 2 (1991), no. 4, 829-860.

[E] B. Enriquez, A cohomological construction of quantization functors of Lie bialgebras, Adv. Math. 197 (2005), no. 2, 430-479.

[EE] B. Enriquez and P. Etingof, On the invertibility of quantization functors, J. Algebra 289 (2005), no. 2, 321-345.

[EG1] P. Etingof and S. Gelaki, On cotriangular Hopf algebras, American Journal of Mathematics 123 (2001), 699-713.

[EG2] Classification of finite-dimensional triangular Hopf algebras with the Chevalley property, Mathematical Research Letters 8 (2001), 249-255.

[EK1] P. Etingof and D. Kazhdan, Quantization of Lie Bialgebras, I, Selecta Mathematica 2 (1996), Vol.1, 1-41.

[EK2] _ Quantization of Lie Bialgebras, II, III, Selecta Mathematica 4 (1998), 213-231, 233269.

[EK3] _ Quantization of Poisson algebraic groups and Poisson homogeneous spaces, Symetries quantiques (Les Houches, 1995), North-Holland, Amsterdam, 1998, pp. 935-946.

[EO] P. Etingof and V. Ostrik, Finite tensor categories, Mosc. Math. J. 4 (2004), no. 3, 627-654.

[ES] P. Etingof and O. Schiffmann, Lectures on quantum groups. Second edition. Lectures in Mathematical Physics. International Press, Somerville, MA, 2002.

[LV] W. T. Lowen and M. Van den Bergh, Deformation theory of abelian categories, to appear in Trans. Amer. Math. Soc., arXiv:math/0405226.

[M] M. Movshev, Twisting in group algebras of finite groups. (Russian) Funktsional. Anal. i Prilozhen. 27 (1993), no. 4, 17-23, 95; translation in Funct. Anal. Appl. 27 (1993), no. 4, 240-244 (1994).

[S] M. Sweedler, Hopf algebras with one grouplike element, Transactions of the American Mathematical Society, 127, No. 3 (1967), 515-526.

Department of Mathematics, Massachusetts Institute of Technology, Cambridge, Ma 02139, USA

E-mail address: etingof@math.mit.edu

Department of Mathematics, Technion-Israel Institute of Technology, Haifa 32000 , ISRAEL

E-mail address: gelaki@math.technion.ac.il 